\title{
Towards a Framework for Health IT Standardization in Mexico
}

\author{
Arturo Serrano-Santoyo ${ }^{1}$, Veronica Rojas-Mendizaba $1^{1}$, Roberto \\ Conte-Galvan ${ }^{1}$, Amanda Gomez-Gonzalez ${ }^{2}$ and \\ Angelica Baptista Silva ${ }^{3}$
}

${ }^{1}$ CICESE Research Center, Ensenada, Baja California, Mexico,

serrano@cicese.edu.mx,vrojas@cicese.edu.mx,conte@cicese.m

${ }^{2}$ Mexican Space Agency, gomez.aem@gmail.com

${ }^{3}$ Oswaldo Cruz Foundation, Brasil, silva.angelica@gmail.com

Received: 29 March, 2013; Accepted: 8 October, 2013

\begin{abstract}
The increasing penetration of ICT in the health sector, in conjunction with the explosion of converging applications covering biotechnology, nanotechnology and green technologies, is creating important opportunities for developing efficient systems and processes that can improve the quality and coverage of health services in developing countries. The pace of technology growth and the lack of comprehensive technology adoption programs and a sound regulatory environment present important challenges if these countries are to take full advantage of the new international scenario of digitalization and global convergence. The role of standardization in a complex and highly converging context is particularly relevant. In consequence, national ICT regulatory administrations in emerging economies are committed to consolidating and strengthening their standardization processes and policies, and national health institutions acknowledge the need for standards that include information technology elements in medical practices. This article delineates the basic elements for the development of a comprehensive framework for Health IT standardization in Mexico. We take into account the multidimensional and complex nature of the standardization process and the importance of interactions among all the actors involved. Our proposal stems from the establishment of a National
\end{abstract}

Journal of ICT, Vol. $3 \& 4,347-362$.

doi: 10.13052/jicts2245-800X.135

(c) 2014 River Publishers. All rights reserved. 
Program for eHealth in Mexico as recommended by both the WHO and the ITU.

Keywords: Health IT, standardization, innovation, ICT regulation.

\section{Introduction}

Information and communications technologies (ICT) play a pivotal role in the conformation of health ecosystems worldwide. The delivery of health services, the implementation of data security and privacy programs and the development of medical devices and future services greatly depend on the deployment of efficient cyber infrastructures. Since the last decade, joint and independent efforts on the part of the World Health Organization (WHO) and the International Telecommunications Union (ITU) have resulted in a series of recommendations to all countries regarding the integration of ICT in their national health information systems and health infrastructure [1,2]. The aim is to support member states with the vision and resources for the development or revitalization of their Health IT policies and strategies, focusing on key emerging technologies such as mobile health (mHealth), telemedicine, eLearning, management of patient information, legal frameworks, safety and security on the internet. Both the WHO and the ITU consider Health IT standardization and interoperability issues as fundamental components for accomplishing a sound national strategy for Health IT, urging member states to structure roadmaps for the implementation of health data standards and to ensure compliance with and adoption of such standards in the public and private sectors [3].

Recognizing the existing disparity between developed and developing countries in relation to standards development, the ITU has focused special attention on reducing this divide. To this aim, the program "Bridging the Standardization Gap" was established in 2009 [4], one objective of which is to identify and understand the gaps that inhibit the process of standards development and implementation in developing countries. At the same time, the ITU acknowledges that the standards capability of a country represents a significant element affecting the digital divide between developed and developing nations.

In light of the above-mentioned concerns, reviewing and upgrading public policies of ICT standardization in developing countries is essential for accelerating their transit toward the knowledge economy, while a diagnosis of the national standards capacity is an important element for assessing and 
identifying each country's primary standardization gaps. As part of the "Bridging the Standardization Gap" program, the ITU developed the Tool for Assessing Standards Capability (TASC) to evaluate national standards readiness in the developing world [5]. The application of this tool provides key indicators for the development of recommendations and best practices for capacity building as well as for the establishment of public policies geared to reducing the existing standardization gap.

Health IT standardization calls for diverse and tailored solutions for each country, involving complex and multidimensional issues in which the understanding and interplay of health, trade and intellectual property are necessary for sound policy making. Likewise, a balance between regulation and innovation is conducive to the configuration of public policies that capitalize on the opportunities for cross-fertilization among the areas involved in Health IT standardization. In this scenario, it is important to acknowledge that innovation in health technologies differs from innovation in general due to the ethical dimension of medical research, the national health regulatory

framework and the liabilities, costs and risks involved [6]. Consideration of these issues entails an interdisciplinary approach in the development of a comprehensive Health IT standardization framework.

Throughout the article we use a conceptualization of standardization as the process of creating, implementing or using a standard (a concept or realization based on common agreements or rules based on set theory) [7]. From this perspective, we consider the case of Health IT standardization, covering the use of ICT in health information systems, infrastructures, equipment, devices, processes, and the delivery of health services. This paper is organized as follows: Section 2 presents a summary of the Mexican Health IT context, highlighting some key indicators, issues and challenges; Section 3 describes the elements of a framework for Health IT standardization in Mexico with reference to the WHO and ITU guidelines and the current policies of health agencies in the Mexican health ecosystem; in Section 4, we identify major challenges that, in our view, Mexico-as well as other emerging economies - faces to develop a sound and sustainable Health IT standardization framework. Our final remarks and conclusions are presented in Section 5 .

\section{The Mexican Context of Health IT Standardization}

With a population of around 114.9 million and a GDP of 1.153 trillion (US dollars), Mexico is the second largest economy in Latin America 
and member of the G20 since 2008 [8]. Given its size in the global economy, the strengthening and consolidation of Mexico's regulatory and standardization frameworks could give rise to significant opportunities for improvements in productivity, competitiveness and innovation in key advanced manufacturing and service sectors. Considering the transversal nature of ICT, reducing the current standardization gap emerges as a significant step towards positioning the country as an important player in the global economy.

With respect to the Mexican health sector, government agencies have made significant efforts to implement strategies and actions that would increase the role of ICT in practically all the links of the value chain of the national Health IT ecosystem $[9,10]$. Furthermore, the ICT regulatory bodies have acknowledged the importance of standardization for advancing the competitiveness indicators of the country; at the same time, the national health institutions recognize the need for standards that include ICT in their medical practices, delivery of services and equipment.

Current governmental and legal structures create a foundation for the development of a comprehensive Health IT standardization framework. The following initiatives are noteworthy:

- Establishment of a national platform for standardization in the health sector, comprising agencies, instruments for medical attention, processes and capacity building [11]. The General Directorate of Quality and Health Education, an agency of the Mexican Ministry of Health (SSA-acronym in Spanish), is responsible for coordinating efforts on health standardization processes in the country (see Figure 1).

- Creation of the National Center for Health Technology Excellence (CENETEC-acronym in Spanish). This Center was created under the auspices of the SSA to produce and disseminate information and the use of medical technologies, based on evidence of their safety, effectiveness and efficiency, for the benefit of the population and the advancement of medical practice [12].

- Publication of two key regulatory instruments: The Mexican Electronic Health Records Standard and the country's Federal Law for Personal Data Protection [13, 14].

- Establishment and strengthening of the E-Mexico National System. This national program was created in December 2000 to propel the country toward the information society [15]. The program is coordinated by the Mexican Ministry of Communications and Transport (SCT-acronym in Spanish). 


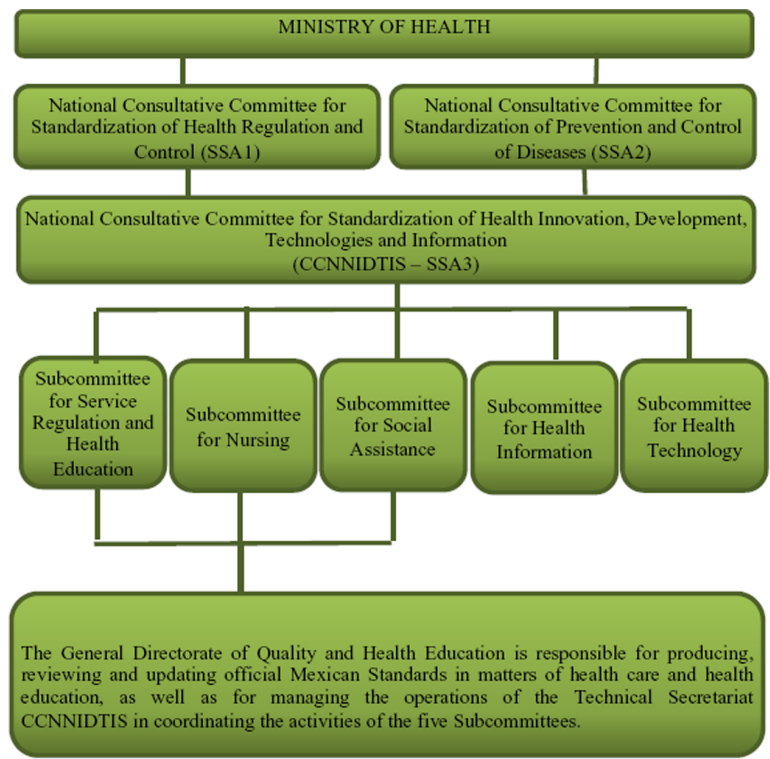

Figure 1 Mexican Health Standardization Structure (adopted from [11]).

Despite the efforts of government and private entities to support, increase and systematize the use of ICT in the health sector, Health IT standardization matters require more sustained attention and strategic actions. The following is a general overview of issues related to the current status of Health IT standardization:

- Results of the application of the ITU-TASC tool for assessing standardization capabilities indicate that the country faces major challenges in standardization matters [5].

- The e-Health country profile for Mexico of the WHO Global Observatory for e-Health shows a significant deficit in key indicators, particularly those related to Health IT standardization [16].

Mexico has a considerable foundation from which it can advance to a more comprehensive and focused participation in Health IT standards development. The strategies and current regulatory structure of the health sector, however, reflect an orientation geared towards simply meeting technology users' needs for standardization. That is, there is a lack of focus on the innovation and technology development that could boost local participation in the creation of standards related to systems, processes and next generation medical devices (including mHealth). Although there is a substantial platform of standards 
covering different areas of medical practice, Health IT standards development requires concentrated strategies and actions in order to reduce the gap and improve the status of Health IT standardization in the country. More important, however, is that key actors in the Mexican health, ICT and regulatory environments acquire an understanding and awareness of the complex and interdisciplinary nature of standardization and its crucial role in the global scenario.

\section{Elements of a Health IT Standardization Framework for Mexico}

The pressing need for improving the quality and coverage of medical services in developing countries, particularly in remote and under-served locations, represents an important opportunity for the development of a comprehensive framework for Health IT standardization. We argue that this framework should adopt an interdisciplinary and collaborative approach and that Mexican health and ICT regulatory bodies need to take into account the importance of the development of endemic Health IT standards rather than merely focusing on their use and implementation. Moreover, we also posit that the process of standards creation emerges from the interactions of the four subsystems forming the Health IT ecosystem. This ecosystem can be viewed as an open socio-technical-legal system. Figure 2 shows the four subsystems considered

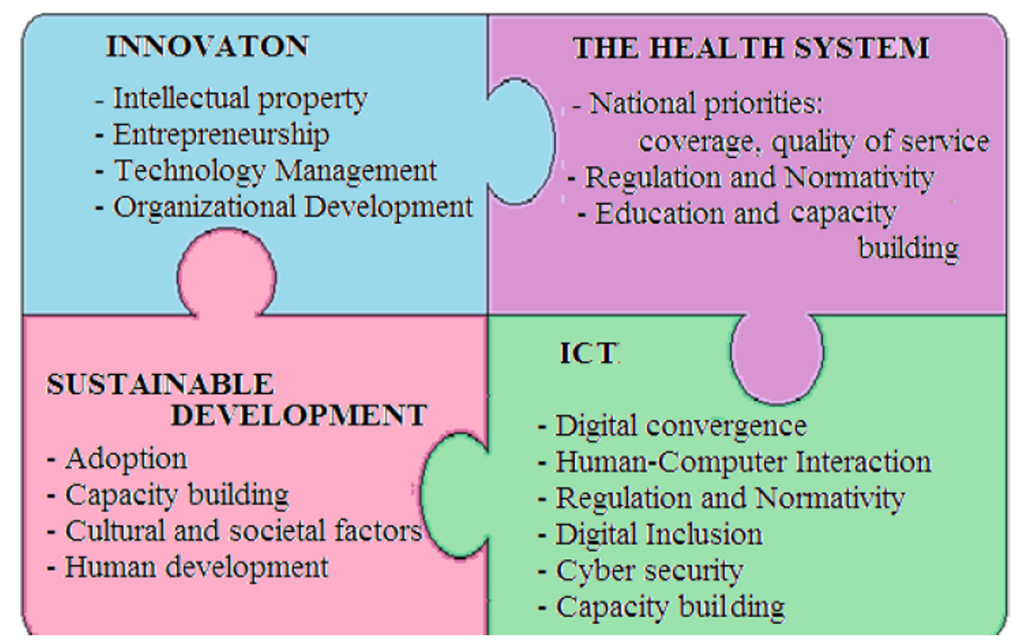

Figure 2 The Health IT Ecosystem. 
in our approach: the innovation subsystem, the national health subsystem, the sustainable development subsystem and the ICT subsystem.

We also believe that a sound Health IT standardization process requires strategic and integrated action at the national level, making use of existing capacity while providing a solid foundation for investment and innovation, as suggested in the National eHealth Strategy Toolkit [2] developed jointly by the WHO and the ITU. We maintain that the application of this resource requires the interaction of actors with experience in strategic planning, communication and collaborative work.

Figure 3 shows the components of the National eHealth Strategy Toolkit and identifies the critical steps in each one of its three stages. The use of this Toolkit and the end result depend on the unity of vision of all stakeholders in regard to the critical issues involved in Health IT standardization and the successful orchestration of interactions among the subsystems described in Figure 2. The World Health Organization uses the term eHealth, which we consider to be the equivalent of the term employed by the ITU, Health IT. In this paper we have adopted the ITU term, Health IT, and only use the term eHealth for referencing purposes.

The National Health IT strategy for Mexico functions as the foundation for the development of our proposal. In our view, a program for Health IT standardization should arise from a sound national strategic plan with the participation of governmental, industrial and academic stakeholders. We

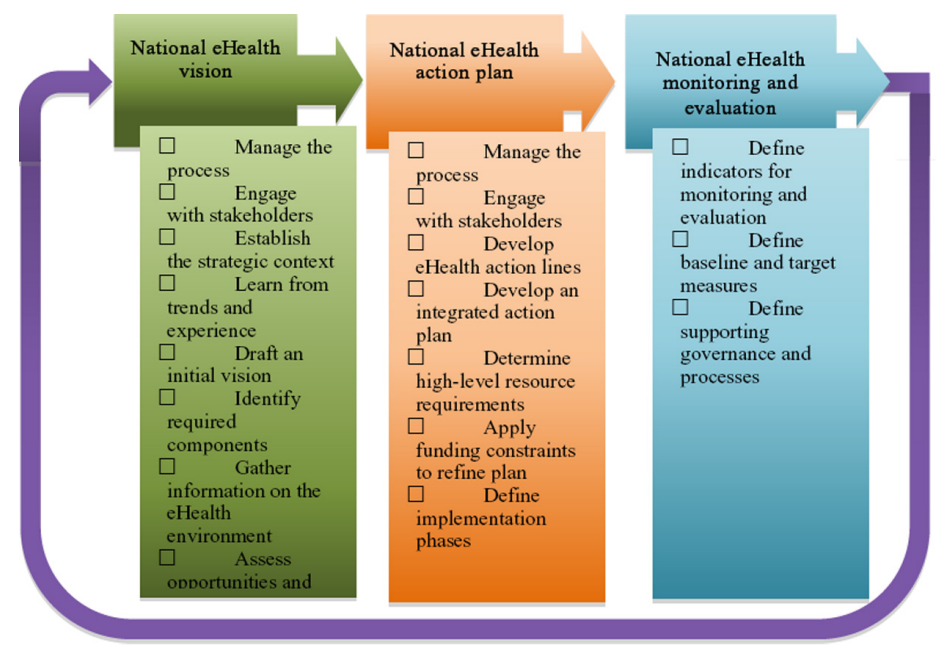

Figure 3 Toolkit for developing a National eHealth Strategy (adopted from [2]). 


\section{Arturo Serrano-Santoyo et al.}

propose concrete steps for the development of a framework for Health IT standardization; Figure 4 describes the elements of our approach. We build our proposal around the creation and use of flexible standards [17] that can adapt to the frequent changes that are characteristic of the complex health environment. The flexible standards approach has demonstrated its usefulness when applied in various developing countries. Viewed from this perspective, standards can be created and maintained as complex adaptive systems where flexibility in use and scalability are essential for achieving the purpose of the standard. The authors of this flexible standards strategy argue that standards must be simple and easy to change and at the same time support a wide range of work practices, thus enabling radical change through small steps. Based on the flexible standards strategy, we propose that the process of Health IT standardization in Mexico initiate with the selection of one particular priority standard. The experience gained in the implementation of this standard would allow us to scale higher levels of complexity, including other standards and other requirements of the Health IT ecosystem as suggested by the above-mentioned authors [17].

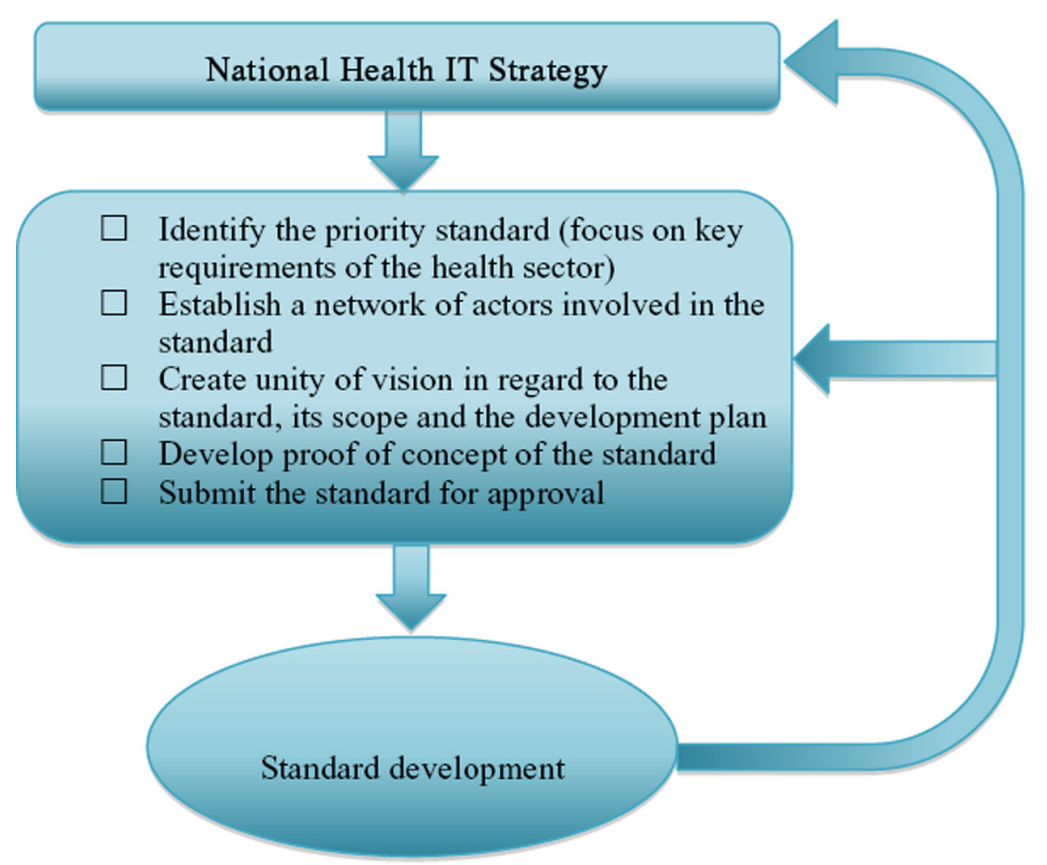

Figure 4 Elements of a framework for Health IT standardization. 


\section{Identifying Major Challenges in the Development of a Health IT Standardization Framework}

As was mentioned in Section 2, CENETEC is a key institution in the Mexican Health IT ecosystem. CENETEC oversees the standardization of medical equipment and systems involving the use of technology. It is therefore the de facto organism in charge of Health IT standardization in the country. Since its inception in 2004, CENETEC has been the liaison with international standards bodies and has participated in the development of important documents for the WHO and other authorities working in Health IT and related fields [18, 19]. CENETEC's contributions to standardization matters in Mexico can be summarized as follows:

- Development of Mexican normativity (NOM, Mexican Official Standards) for:

a) Generic medical instruments

b) Medical devices

c) Infrastructure and equipment requirements for medical facilities

- Development of guidelines for technical specifications.

- Development of guidelines and models for medical equipment.

- Development of guidelines for the implementation of Telehealth projects (infrastructure and equipment)

- Development of guidelines for clinical practices

According to CENETEC [20], it is necessary to address priority issues in the conformation of a sound and comprehensive standardization framework for Health IT. Some of the issues and recommendations are:

- Intensify the scope and coverage of standardization in all healthcare services of public and private medical institutions.

- Identify the cost-benefit, cost-impact and cost-opportunity indicators for Health IT.

- There is a widespread lack of "digital skills" and "informatics and telecommunications culture" at all levels of the Health IT ecosystem. This condition applies to medical practitioners as well as to decision-makers and management personnel.

- Some medical practitioners consider emerging technologies as a threat.

- There is a lack of a legal and ethics framework for Health IT.

The authors acknowledge the important work and contributions of CENETEC to the Mexican Health IT context. We suggest that in order to capitalize on 
the benefits of Health IT as well as achieve a comprehensive participation of Mexican institutions in standards creation, some remaining barriers should be eliminated:

- Infrastructure in rural and in some suburban locations is largely insufficient to utilize the most current Internet technologies.

- Even when basic infrastructure is in place, widespread interoperability standards for software are lacking.

- Local skills, knowledge, and resources may also limit the application of Health IT devices, equipment and systems.

- Failing to account for incompatible cultural subsystems that prevent the transfer of knowledge from one cultural context to another is a major contributing factor to Health IT failure. Without a good understanding of the local context, it may be difficult to integrate Health IT in a useful way.

Additionally, we posit that in order to develop a national program for Health IT standards creation, the consideration of the following factors is essential:

1. Development of a detailed analysis of the Mexican Health IT ecosystem including the interactions of the major agents in the strategic, tactic and operational levels.

2. Alignment of the national program for Health IT standardization in Mexico with the WHO strategic plan for 2012-2017 [21] as described in Figure 3.2.

With respect to the Mexican Health IT ecosystem, we acknowledge additional challenges such as:

1. The aspect of culture: Emerging economies such as Mexico face challenges in their efforts to increase competitiveness and productivity indicators. As mentioned above, a sound standardization framework is a key factor for strengthening the national regulatory environment, at the same time providing an opportunity for interaction between government and academia. Such collaboration can lead, as in the case of Korea [22], to the actors working together in building a national "culture of standardization" that permeates all strata of society. The development of this culture requires a long-term vision with strategic, tactical and operational stages that include the participation of all stakeholders. Furthermore, reducing the standardization gap in a comprehensive manner calls for the adoption of an interdisciplinary approach. 
2. Capacity building: An essential element for the deployment of a comprehensive national standardization plan, capacity building is particularly relevant for Health IT and related emerging disciplines. The importance of capacity building is evident when one considers that it is required for three of the four aspects of the Health IT ecosystem (see Fig. 3.1). Academic institutions play a key role in the design and implementation of educational and training programs that stress the technical, legal and innovation issues involved.

3. Learning by doing: The participation of Mexican specialists in ITU standardization working groups and in other international standards agencies would significantly contribute to the development of the skills and practical knowledge required in all the links of the Health IT value chain. Achieving this participation would, of course, require allocation of the necessary funds.

\section{Final Remarks and Conclusions}

Based on the analysis presented in Sections 2, 3 and 4, we identified major areas that should be addressed in order to improve the status of Health IT standardization in Mexico:

- The need to establish a national Health IT policy emphasizing the central role of standardization in the value chain of medical processes and technologies. The framework for Health IT standardization would be a key component of this national policy.

- Given the nature and dynamics of the Health IT ecosystem, the legal and ethical aspects of the framework are of particular importance and must be addressed.

- Capacity building and the establishment of a national program that permeates all levels of society are two critical aspects for fostering a culture of standardization in the country [22].

- As suggested by the WHO [16], the Health IT standardization framework should take into account the following disciplines: telemedicine, mHealth, eLearning in health sciences for all the medical specialties, management of patient and medical infrastructure, as well as the legal and ethical elements involved.

- In order to fully capitalize on interoperability, it is important to focus on patients' needs and their interaction with data and technology. In this respect, patient education and the departure from a fragmented and technology centric perspective are essential if we are to take advantage 
of the full potential of Health IT. Our approach, which conceptualizes the Health IT ecosystem as a socio-technical-legal system, would support the development of an integrated and all-inclusive national Health IT plan.

- A perspective that is informed by complexity theory, as suggested in the flexible standards strategy [17], constitutes a pragmatic and integral approach for standards creation in developing countries. Complexity theory is proving to be relevant for understanding and explaining the challenges and needs of a sustainable Health IT framework.

An integral framework for Health IT standardization could provide a catalyst for moving away from a condition of mere technology usage toward a technology development model. Embracing this transition would allow, in the long term, the deployment of cost-effective medical services and products in order to meet the varying needs of citizens and health institutions.

Without a comprehensive framework for Health IT in developing countries, achieving the application of ICT as a vehicle for human development will remain elusive and an important opportunity to participate in the definition and creation of standards of global impact will be lost. The experiences of developing countries in the provision of Health IT services in rural and underserved areas are very important, and the development of a national standards framework for Health IT that is supported by all the regulatory agencies involved is, in our view, a fundamental step in the advancement of Mexico toward the knowledge society. Rather than defining or recommending the development of a particular standard or set of standards, our purpose in this article has been to propose a starting platform for Health IT standardization. We recognize that it is necessary to develop a strategic and tactical plan with its associated lines of action in which an approach from complexity science could contribute to understanding the context and interactions of all the actors involved in the Mexican health sector.

\section{References}

1 eHealth and Health Internet Domain Names. Report by the Secretariat, World Health Organization, 11 January 2013.

2 National eHealth Strategy Toolkit. World Health Organization and International Telecommunications Union. Available at: http://www.who .int/ehealth/en/

3 eHealth standardization and interoperability. WHO $132^{\text {nd }}$ session, Agenda item 10.5, 28 January 2013. 
4 Bridging the Standardization Gap: Measuring and Reducing the Standards Gap, ITU, 4 December 2009.

5 Tool for Assessing Standards Capability (TASC), ITU-T, 4 December 2009.

6 Promoting Access to Medical Technologies and Innovation, WHO-WIPO-WTO. Available at: http:// www.who.int/phi/en/

7 Krechmer, K. "Teaching Standards to Engineers", The International Journal of IT Standards and Standardization Research, Vol 5, No. 2, July-December 2007.

8 World Bank Data: Mexico. Available at: http://www.worldbank.org/coun try/mexico.

9 Manual del Expediente Clínico Electrónico, Dirección General de Información en Salud, Secretaría de Salud, México, 2011.

10 Serie Tecnologías en Salud, Volumen 3 Telemedicina, Secretaría de Salud, México, 2011.

11 Procesos Normativos en Salud, Dirección General de Calidad y Educación en Salud, Secretaría de Salud. Available at: http://www.calidad.sa lud.gob.mx/normatividad/procesos_normativos_salud.html

12 Centro Nacional de Excelencia Tecnológica en Salud, CENETEC. www.cenetec.gob.mx

13 Norma Oficial Mexicana NOM-024-SSA3-2010, Diario Oficial de la Federación, 16 Agosto 2010.

14 Ley Federal de Protección de Datos Personales en Posesión de los Particulares, Diario Oficial de la Federación, 5 Julio 2010.

15 El programa E-México, www.emexico.gob.mx

16 Global Observatory for eHealth, WHO, 2009. Available at: http://www .who.int/goe/data/en/

17 J. Braa et al, "Developing Health Information Systems in Developing Countries: The Flexible Standards Strategy", MIS Quarterly Vol. 31 Special Issue, pp. 1-22, August 2007.

18 Telemedicine. Opportunities and developments in Member States, Report on the second global survey on eHealth, Global Observatory for eHealth series, Volume 2, WHO, 2010.

19 N. Gertrudiz, "e-Health: the case of Mexico", Latin Am J Telehealth, Vol 2, No. 2, pp 127-167, 2010. 
20 A. Velazquez, "The landscape of e-Health issues in Mexico" ETSI Meeting on eHealth, Sophie Antipolis 2007.

21 eHealth Strategy and Plan of Action (2012-2017), PAHO/WHO, 2011

22 Bum H. K. et al, Future Society \& Standards, Korean Standards Association, 2004/2007.

\section{Biographies}

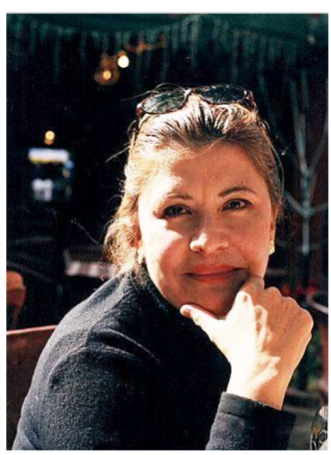

AMANDA O. GOMEZ-GONZALEZ Dr. GomezGonzalez got her PhD in telecommunications. She has more than 20 years of experience regarding national and Latin American programs in telemedicine, telehealth and e-Health. She was founding director of the Mexican National Telehealth Programme. Dr. Gómez-González is currently governmental development manager of the Mexican Space Agency and Professor at the National Autonomous University of Mexico, UNAM.

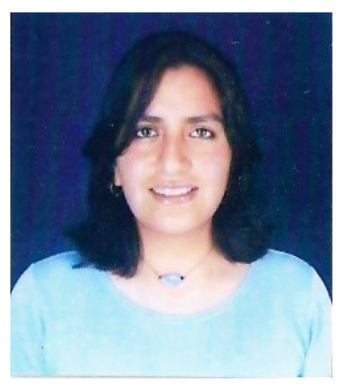

VERONICA ROJAS-MENDIZABAL Veronica Rojas-Mendizabal is a Telecommunications Engineer. She earned her Master's degree in Telecommunications and Telematics from the Catholic University of Bolivia (UCB) in La Paz, Bolivia in 2008. She collaborated with the Regional Center for Education in Space Science and Technology for Latin America and the Caribbean (CRECTEALC), a United Nations space educational center, in Puebla, Mexico. She is currently a doctoral student in Electronics and Telecommunications at CICESE Research Center in Ensenada, Baja California, Mexico. 


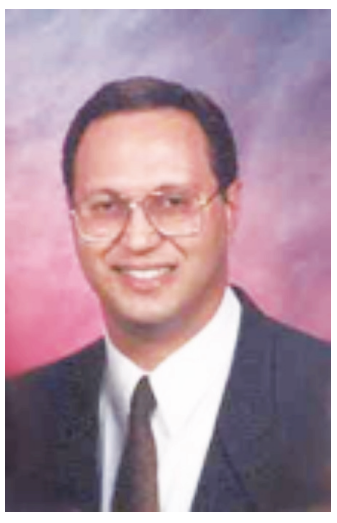

ROBERTO CONTE-GALVAN Dr. Roberto ConteGalvan got his Ph.D. in Electrical Engineering from Virginia Polytechnic Institute \& State University (Virginia Tech, USA) in 2000. He coordinated the first Gradute course on Telemedicine in Mexico and Latin America. He is currently a Full Researcher at the Department of Electronics and Telecommunications and member of the Telecommunications Networks Group at CICESE Research Center in Ensenada, Baja California, Mexico. Dr. Conte serves as representative and executive liaison with the Mexican Space Agency.

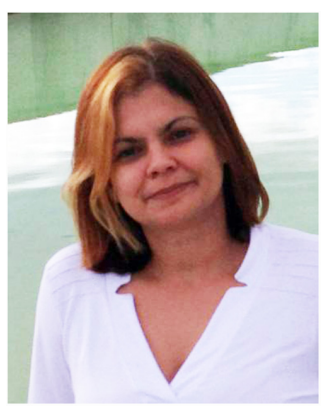

ANGELICA BAPTISTA SILVA Dr. Angelica Baptista Silva earned a PhD in Public Health in 2013. She is a specialist in Internet with a degree in Social Communication and Journalism. She was coordinator of the Health TV Channel at the Department of Information Technology of the Oswaldo Cruz Foundation/FioCruz from 1998-2011 and since 2012 coordinates the activities of the Telehealth Laboratory of the National Institute of Women, Children and Adolescent's Health Fernandes Figueira (IFF / FioCruz) and the International Network of Human Milk Banks. She is currently member of the Advisory Committee and coordinator of the Content Usage Working Group at the Brazilian Telemedicine University Network/RUTE.

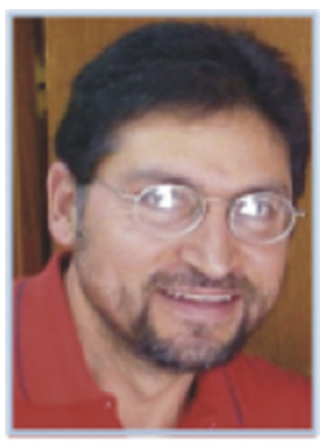

ARTURO SERRANO-SANTOYO Dr. SerranoSantoyo earned his Doctor's degree in Electrical Engineering from the National Polytechnic Institute in Mexico City in 1980. In 1981 he received the ALCATEL Annual Telecommunications Award for his contributions to rural satellite communications in Mexico and Latin America. He has been a telecommunications consultant for the Organization of American States and the United Nations, as well as for many private companies and governmental agencies. He is currently researcher at CICESE Research Center in Ensenada, Baja California, Mexico and Professor at the Autonomous University of Baja California. 
